\title{
Pretherapy Gallium-67 Scanning in Paediatric Patients with Hodgkin's Disease
}

\author{
G.M. Shah Syed ${ }^{a}$ M.N. Younis ${ }^{a} \quad$ G.N. Usmani ${ }^{b} \quad$ N. Zafar ${ }^{c}$ \\ a Department of Nuclear Medicine, Faculty of Medicine, Kuwait University, Kuwait; \\ bShaukat Khanum Memorial Cancer Hospital and Research Centre, Lahore, Pakistan
}

\section{Key Words}

Hodgkin's disease, subtypes • Gallium-67 scanning •

Gallium-67 uptake

\begin{abstract}
Objective: To investigate the correlation between gallium-67 ( $\left.{ }^{67} \mathrm{Ga}\right)$ uptake and histological subtypes of Hodgkin's disease (HD) in paediatric patients. Subjects and Methods: Fifty-eight patients (45 males and 13 females aged $9.2 \pm 4$ years, range 1.5-17 years) with histologically diagnosed HD underwent pretherapy ${ }^{67} \mathrm{Ga}$ scanning on days 2, 5, 12 or 14 days after intravenous administration of $25-50 \mathrm{MBq}(0.7-1.4 \mathrm{mCi})$ of ${ }^{67} \mathrm{Ga}$ citrate. The scans were evaluated both visually and quantitatively using the activity of ${ }^{67} \mathrm{Ga}$ in the liver as a reference. Clinical outcome of 11 patients with high diffuse ${ }^{67} \mathrm{Ga}$ skeletal uptake was compared with that of 17 patients showing normal distribution of ${ }^{67} \mathrm{Ga}$ in the skeleton. Results: Of the 58 patients, the ${ }^{67} \mathrm{Ga}$ scans were positive in 47 patients with 117 lesions. Visual analysis did not differentiate between the histological variants of HD. However, quantitative analysis of lesion-to-liver ratios showed significantly higher values of ${ }^{67} \mathrm{Ga}$ uptake in the mixed cellularity type than in the nodular sclerosis type $(t=-3.7, p<0.001)$. Patients with high skeletal uptake had
\end{abstract}

\section{KARGER}

Fax +41613061234

E-Mail karger@karger.ch

www. karger.com
(C) 2004 S. Karger AG, Basel

1011-7571/04/0132-0074\$21.00/0

Accessible online at: www. karger.com/mpp a higher relapse rate $(6 / 11)$ than those with normal skeletal uptake (3/17). Conclusion: The findings show that quantitative analysis of ${ }^{67} \mathrm{Ga}$ uptake can differentiate between the two main subtypes of HD (mixed cellularity and nodular sclerosis). Further diffuse skeletal uptake of ${ }^{67} \mathrm{Ga}$ indicates a higher relapse rate.

Copyright $@ 2004$ S. Karger AG, Basel

\section{Introduction}

Hodgkin's disease (HD) is a malignant neoplastic disorder primarily involving the lymphoid tissue and is characterized by the presence of distinct giant cells called Reed-Sternberg cells, admixed with a variable inflammatory infiltrate. Four subtypes of HD are well recognized: lymphocyte predominance (LP), mixed cellularity (MC), lymphocyte depletion (LD) and nodular sclerosis (NS). Treatment strategies depend primarily on the histological subtype and other factors that include the presence of $\mathrm{B}$ symptoms, gender, and sexual maturity [1].

Although positron emission tomography using fluorine-18-deoxyglucose is a highly sensitive and specific imaging modality for the management of HD [2], Gallium-67 $\left({ }^{67} \mathrm{Ga}\right)$ whole-body scanning has been recognized as a reliable and widely available imaging modality for
G.M. Shah Syed, Ass. Prof. and Consultant Nuclear Physician

Department of Nuclear Medicine, Faculty of Medicine

Kuwait University, PO Box 24923

13110 Safat (Kuwait)

Tel. +965 4815578, Fax +965 5318454, E-Mail gmshahsyed@hotmail.com 
accurate diagnosis of residual disease by differentiating it from fibrosis or necrotic tissue [3, 4]. Further ${ }^{67} \mathrm{Ga}$ scan is used for evaluation of response to therapy, detection of relapse and prediction of disease-free survival in posttherapy patients with HD [3-6]. Heterogeneity of ${ }^{67} \mathrm{Ga}$ uptake has been shown in histological subvarieties of HD [6]. Efforts have been made earlier to study the correlation between ${ }^{67} \mathrm{Ga}$ uptake and the histological subtypes of HD [7-9]. However, these studies were largely based on visual interpretation and showed poor correlation between the histology and ${ }^{67} \mathrm{Ga}$ uptake. This work was undertaken to study the correlation between ${ }^{67} \mathrm{Ga}$ uptake and the histological subtypes of HD in paediatric patients using a quantitative method.

\section{Subjects and Methods}

Fifty-eight paediatric patients histologically diagnosed with HD were included in the study. Forty-five patients were males and 14 females. Mean age at presentation was $9.2 \pm 4$ years (1.5-17 years). Demographic data and histopathological subtypes were obtained from patients' records: 44 patients had MC, $9 \mathrm{NS}, 2 \mathrm{LP}$ and $1 \mathrm{LD}$ type. The histological subtype of HD was not established in 2 patients despite using immunohistochemical staining technique.

\section{${ }^{67}$ Ga Scanning}

${ }^{67} \mathrm{Ga}$ scanning was performed in all patients prior to Chemotherapy. A dose of $1.7 \mathrm{MBq}(0.05 \mathrm{mCi}) / \mathrm{kg}$ body weight of ${ }^{67} \mathrm{Ga}$ citrate (M/S Amersham International, PLC, UK) with an average of $37 \mathrm{MBq}(1.0 \mathrm{mCi}$; range $25-50 \mathrm{MBq}, 0.1-1.35 \mathrm{mCi})$ was injected intravenously. Images were acquired on days 2, 5 and 12 or 14 in all cases. The patient was advised to take a mild purgative (Dulcolax) on the night prior to imaging on all occasions. Each patient was positioned supine under the gamma camera (Diacam, M/S Siemens). Scanning was performed using high energy collimator multiple energy window settings $(93,184$ and $296 \mathrm{keV})$ and a zoom factor of 1.23 except for large patients. Multiple, overlapping, digital static images were acquired by computer in $128 \times 128$ word mode matrix. Each view was acquired for a total of 200,000 counts or $20 \mathrm{~min}$, whichever came first. On days 2 and 5 after injection, images were acquired in the anterior and posterior projections to include head, neck, chest, abdomen and pelvis. All patients were scanned either on day 12 or 14 for views of the abdomen and pelvis. Images were recalled and displayed in grey scale. Hard copy was developed on single-coated 810inch X-ray film.

Interpretation of ${ }^{67} \mathrm{Ga}$ Images. Scans were interpreted by 2 nuclear physicians by consensus. The following patterns of ${ }^{67} \mathrm{Ga}$ accumulation were reported as abnormal: (a) accumulation in cervical, mediastinal, axillary and inguinal regions, (b) patchy and nonuniform accumulation in the liver or spleen, (c) increased accumulation in the abdomen and pelvis that did not change in position over the 12 or 14 days, and (d) uptake in bones equal to or higher than the liver. Each area of abnormal ${ }^{67} \mathrm{Ga}$ accumulation was graded relative to liver activity as follows: lesion less than the liver but higher than background $=$ grade 1 ; equal to the liver $=$ grade 2 , and higher than the liver $=$ grade 3 .

${ }^{67} \mathrm{Ga}$ Uptake and Subtypes of Hodgkin's

Disease
Table 1. Results of ${ }^{67} \mathrm{Ga}$ scanning in various histological subtypes of HD

\begin{tabular}{lrllll}
\hline & MC & NS & LP & LD & US \\
\hline Positive ${ }^{67}$ Ga scan & 36 & 9 & 1 & 1 & 0 \\
Negative ${ }^{67}$ Ga scan & 8 & 0 & 1 & 0 & 2 \\
\hline Total & 44 & 9 & 2 & 1 & 2 \\
\hline
\end{tabular}

US = Unspecified, where subtype of HD could not be established.

Quantification. ${ }^{67} \mathrm{Ga}$ uptake was quantified to obtain lesion-toliver uptake ratio (LLR). Using a region ratio protocol available in processing options of the software, regions of interest (ROIs) were drawn on the lesion, liver and background. The ROI was drawn on the lesion first by encircling the centre of the lesion. An equal pixel size liver ROI was obtained through duplication of lesion ROI. The background ROI was drawn in the soft tissue above the clavicle on the disease-free side. In cases of bilateral cervical disease, the ROI for background correction was drawn in the axillary soft tissue avoiding ${ }^{67} \mathrm{Ga}$ activity in the humerus. The software was then used to calculate the LLR using background corrected means of the two ROIs. Means were calculated and statistical analysis was done. Differences between the groups were tested by Student's $t$ test for independent samples.

\section{Results}

Of the 58 patients, $47(81 \%)$ and $11(19 \%)$ had positive and negative ${ }^{67} \mathrm{Ga}$ scans, respectively. Results of ${ }^{67} \mathrm{Ga}$ scanning by histological subtypes of HD are shown in table 1 . The 9 NS patients were all positive while 36 of 44 (82\%) MC patients were positive. The remaining subtypes, LP, LD and unspecified, had only 2, 1 and 3 patients, respectively, and therefore the rate of ${ }^{67} \mathrm{Ga}$ positivity was not calculated. Of the 11 patients with negative ${ }^{67} \mathrm{Ga}$ scan, 3 had a single-site lesion that was excised for histopathological diagnosis.

Cervical lymph nodes were the commonest site of involvement in all four subtypes, followed by mediastinal disease and abdominal involvement (table 2). Of 117 lesions, 78 were of grade 3,33 lesions were of grade 2 and 6 grade 1 (table 3 ).

Quantification of uptake performed in 45 patients showed 107 sites of abnormal ${ }^{67} \mathrm{Ga}$ accumulation. It included all 81 lesions in 36 patients with MC and 26 lesions in 9 patients with NS type. In MC, LLR value was $1.95 \pm 1.04$ (range 0.14-4.79) and in NS type mean LLR value was $1.14 \pm 0.67$ (range $0.11-2.12$ ) and the differ- 

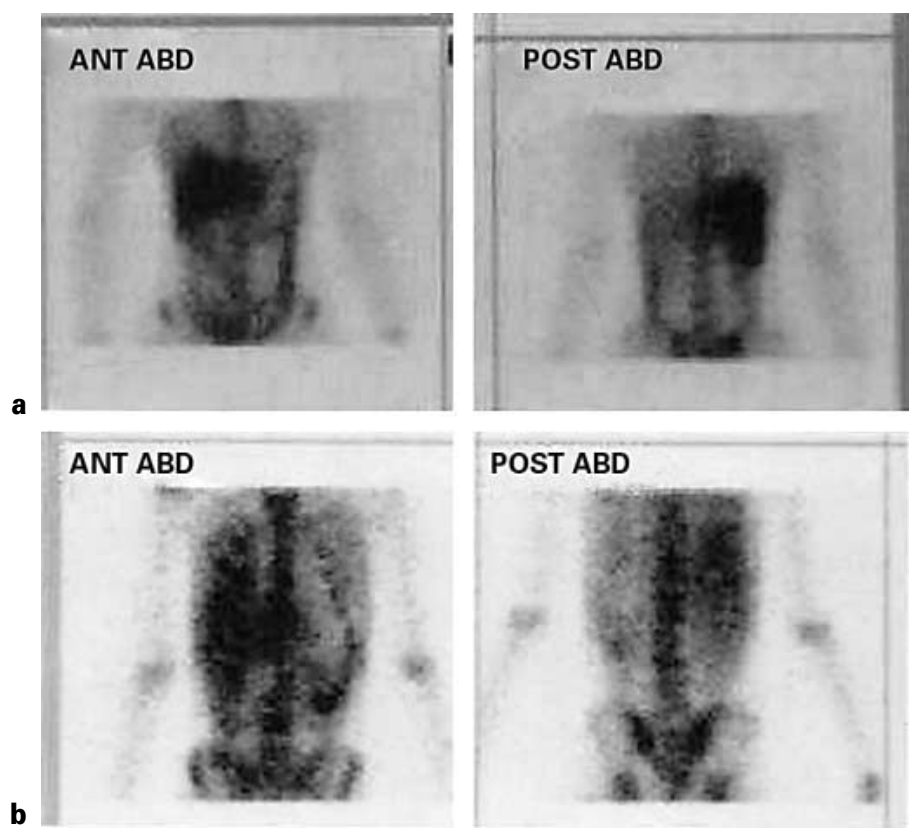

Fig. 1. ${ }^{67} \mathrm{Ga}$ scan of a patient with normal skeletal uptake (a) and high uptake in the skeleton (b). ANT = Anterior; $\mathrm{POST}=$ posterior; $\mathrm{ABD}=$ abdomen

Table 2. Lesion distribution in histological subvarieties of HD in 47 pretherapy patients with positive ${ }^{67} \mathrm{Ga}$ scan

\begin{tabular}{lccccc}
\hline Histology & $\begin{array}{l}\text { Cervical } \\
\text { disease }\end{array}$ & $\begin{array}{l}\text { Thoracic } \\
\text { disease }\end{array}$ & $\begin{array}{l}\text { Abdominal Others } \\
\text { disease }\end{array}$ & $\begin{array}{l}\text { Total } \\
\text { lesions }\end{array}$ \\
\hline MC $(\mathrm{n}=36)$ & 39 & 27 & 11 & 4 & 81 \\
NS $(\mathrm{n}=9)$ & 13 & 9 & 4 & - & 26 \\
LP $(\mathrm{n}=1)$ & 5 & 2 & - & - & 7 \\
LD $(\mathrm{n}=1)$ & 2 & - & 1 & - & 3 \\
\hline Total & 59 & 38 & 16 & 4 & 117 \\
\hline
\end{tabular}

Table 3. Visual grading of ${ }^{67} \mathrm{Ga}$-avid lesions in subtypes of HD histology

\begin{tabular}{llccc}
\hline & Grade 1 & Grade 2 & Grade 3 & Total \\
\hline MC $(\mathrm{n}=81)$ & 5 & 22 & 54 & 81 \\
NS $(\mathrm{n}=26)$ & 1 & 9 & 16 & 26 \\
LP $(\mathrm{n}=7)$ & - & 2 & 5 & 7 \\
LD & - & - & 3 & 3 \\
\hline Total & 6 & 33 & 78 & 117 \\
\hline
\end{tabular}

$\mathrm{n}=$ Number of lesions ence was statistically significant $(p<0.001)$. Also there was a statistically significant correlation between the visual grading and LLR ratios in both subvarieties $(\mathrm{r}=$ $0.87, \mathrm{p}<0.001$ in $\mathrm{MC}$ and $\mathrm{r}=0.87, \mathrm{p}<0.001$ in NS).

Of the 11 patients with high skeletal uptake of ${ }^{67} \mathrm{Ga}$ (equal to or higher than the liver), 3 had a positive bone marrow biopsy for lymphoma infiltration and during a period of 6 months, 6 of them had a relapse. However, in the 17 patients with skeletal ${ }^{67} \mathrm{Ga}$ uptake lower than the liver, none had a positive bone marrow biopsy for lymphoma infiltration and only 3 relapsed in 6 months follow-up. Figure 1a shows a ${ }^{67} \mathrm{Ga}$ scan in a patient with normal skeletal uptake while figure $1 \mathrm{~b}$ represents a patient with high skeletal uptake.

\section{Discussion}

Heterogeneity of ${ }^{67} \mathrm{Ga}$ uptake in various subtypes of HD and non-Hodgkin's lymphoma has been reported in many studies [6]. The subtypes of NS, MC, and LD account for about $90 \%$ of cases that are ${ }^{67} \mathrm{Ga}$-avid [3-5]. LP tumours (10\% of all cases) show somewhat less ${ }^{67} \mathrm{Ga}$ avidity.

In this study, of the $81 \%$ positive ${ }^{67} \mathrm{Ga}$ scans, NS type was highest (100\%), followed by MC ( $82 \%)$, which is similar to previous studies where the sensitivity of the ${ }^{67} \mathrm{Ga}$ scan had been reported for MC and LD to be $90 \%$ followed by: NS $85-89 \%$ and LP $79 \%$ varieties $[11,12]$. Unfortunately due to the small number of patients with LD, LP and US subtypes in this study, a meaningful rate of ${ }^{67} \mathrm{Ga}$ positivity was not determined for these subtypes and therefore, we cannot compare our results with those already reported in other studies. High skeletal uptake of ${ }^{67} \mathrm{Ga}$ has been observed in various pathologies that include hyperplasia [13], leukaemia, lymphoma and HIV infection $[14,15]$ as seen in 11 patients of the present study. The mechanism is not quite obvious but it has been explained variously as due to the state of iron metabolism $[8,13]$ in pathological conditions that reflect involvement of bone marrow by the disease process $[14,15]$. In this study 6 of the 11 patients with high skeletal ${ }^{67} \mathrm{Ga}$ uptake had a relapse when compared to 3 of 17 with normal skeletal uptake. Serum ferritin levels and other indicators of iron metabolism were within normal range. Therefore, we suggest that the high skeletal ${ }^{67} \mathrm{Ga}$ uptake could be due to extensive bone marrow involvement that ultimately led to the relapse of the disease in 6 patients. To confirm this hypothesis, multiple bone marrow biopsies from various sites would be helpful. 


\section{Conclusion}

The results of this study show that ${ }^{67} \mathrm{Ga}$ scan detected disease in the various histological subtypes of HD, particularly NS and MC, leading to an overall positive rate of more than $80 \%$. Although visual grading of ${ }^{67} \mathrm{Ga}$ uptake was sensitive in detecting disease sites, semi-quantitative analysis was able to identify an association between the degree of ${ }^{67} \mathrm{Ga}$ uptake and histological subtypes $\mathrm{MC}$ and NS. Further, high skeletal uptake of ${ }^{67} \mathrm{Ga}$ might be an indicator of the disease progress.

\section{References}

1 Jenkin D, Doyle J, Berry M, Blanchette V, Chan H, Doherty M, Freedman M, Greenberg M, Panzarella T, Saunders F, et al: Hodgkin's disease in children: Treatment with MOPP and low-dose, extended field irradiation without laparotomy: Late results and toxicity. Med Pediatr Oncol 1990;18:265-272.

2 Menzel C, Dobert N, Mitrou P, Mose S, Diehl M, Berner U, Grunwald F: Positron emission tomography for the staging of Hodgkin's lymphoma: Increasing the body of evidence in favor of the method. Acta Oncol 2002;41:430436.

3 Nikpoor N, Aliabadi P, Diaz L, Mannting F: Long-term follow-up of residual mediastinalhilar Ga-67 uptake after treatment for Hodgkin's and non-Hodgkin's lymphomas: What degree of $\mathrm{Ga}-67$ uptake is significant? Clin Nucl Med 2000;25:959-962.

4 Israel O, Front D, Epelbaum R, Ben-Haim S, Jerushalmi J, Kleinhaus U, Even-Sapir E, Robinson $\mathrm{E}$ : Residual mass and negative gallium scintigraphy in treated lymphomas. J Nucl Med 1990;31:365-368.
5 Front D, Israel O, Epelbaum R, Ben Haim S, Sapir EE, Jerushalmi J, Kolodny GM, Robinson E: Ga-67 SPECT before and after treatment of lymphoma. Radiology 1990;175:515519.

6 Front D, Bar-Shalom R, Epelbaum R, Haim N, Ben-Arush MW, Ben-Shahar M, Gorenberg M, Kleinhaus U, Parmett S, Kolodny GM: Early detection of lymphoma recurrence with Ga-67 scintigraphy. J Nucl Med 1993;34:2101-2104.

7 Front D, Israel O: Present state and future role of gallium 67 in lymphoma. J Nucl Med 1996; 37:530-532.

8 Flores LG 2nd, Jinnouchi S, Nagamachi S, Ohnishi T, Futami S, Watanabe K: Increased bone marrow uptake of Ga-67 in patients with fever of unknown origin. Clin Nucl Med 1996; 21:786-791.

9 Ohzono H: Visual analysis of hepatic function by a scoring system using ${ }^{99 \mathrm{~m}} \mathrm{Tc}-\mathrm{GSA}$. Kaku Igaku 1996;33:935-942.

10 Almer S, Peters AM, Ekberg S, Franzén L, Granerus $\mathrm{G}$, Strom M: Is computer-aided interpretation of ${ }^{99} \mathrm{Tc}^{\mathrm{m}}$-HMPAO leukocyte scans better than the naked eye? Nucl Med Commun 1995; 16:290-298.
11 Bekerman C, Hoffer PB, Bitran JD: The role of gallium 67 in the clinical evaluation of cancer. Semin Nucl Med 1984;14:296-323.

12 Badshah S, Anwar SM, Saleem M: Evaluation of efficacy of an alternating chemotherapy regimen in childhood Hodgkin's disease with evaluation of acute and chronic toxicity (abstract). Proc 2nd Shaukat Khanum Memorial Cancer Symp, 1995.

13 Nguyen J, Owens J, Vasinrapee P, Spreth M: Ga-67 imaging in bone marrow hyperplasia. Clin Nucl Med 1996;21:404-406.

14 del Val Gomez M, Gallardo FG, Cobo J, Castro-Beiras J: Abnormal gallium-67 skull uptake: A sign of peripheral marrow activation in HIV-positive patients with disseminated mycobacterioses. J Nucl Med 1995;36:22112213.

15 Larar GN, Janicek MJ, Kaplan WD: Ga-67 scintigraphy after bone marrow harvest: Significance of 'sacroiliac' asymmetry in the lymphoma patient. Clin Nucl Med 1993;18:126-129. 